\title{
Speckle dynamics under ergodicity breaking
}

\author{
Anton Yu. Sdobnov*,1, Alexander Bykov' ${ }^{1}$, Guillaume Molodij ${ }^{2}$, Vyacheslav Kalchenko², \\ Topias Jarvinen ${ }^{1}$, Alexey Popov ${ }^{1}$, Krisztian $\operatorname{Kordas}^{1}$ and Igor Meglinski ${ }^{13,4}$ \\ ${ }^{1}$ Faculty of Information Technology and Electrical Engineering, University of Oulu, Oulu 90570, Finland \\ ${ }^{2}$ Department of Veterinary Resources, Weizmann Institute of Science, Rehovot 76100, Israel \\ ${ }^{3}$ Interdisciplinary Laboratory of Biophotonics, National Research Tomsk State University, Tomsk \\ 634050, Russia \\ ${ }^{4}$ Institute of Engineering Physics for Biomedicine (PhysBio), National Research Nuclear University \\ MEPhI, 115409 Moscow, Russia \\ * Correspondence: sdobnovanton@mail.ru
}

\begin{abstract}
Laser speckle contrast imaging (LSCI) is a well-known and versatile approach for the noninvasive visualization of flows and microcirculation localized in turbid scattering media, including biological tissues. In most conventional implementations of LSCI the ergodic regime is typically assumed valid. However, most composite turbid scattering media, especially biological tissues, are non-ergodic, containing a mixture of dynamic and static centers of light scattering. In the current study, we examined the speckle contrast in different dynamic conditions with the aim of assessing limitations in the quantitative interpretation of speckle contrast images. Based on a simple phenomenological approach, we introduced a coefficient of speckle dynamics to quantitatively assess the ratio of the dynamic part of a scattering medium to the static one. The introduced coefficient allows one to distinguish real changes in motion from the mere appearance of static components in the field of view. As examples of systems with static/dynamic transitions, thawing and heating of Intralipid samples were studied by the LSCI approach.
\end{abstract}

Keywords: speckle contrast, speckle pattern, motion estimation, optical imaging, ergodicity. 


\section{Introduction}

The spatio-temporal coherence properties of light scattered by rough surfaces and/or by turbid randomly inhomogeneous scattering medium lead to the intensity fluctuations. The image formed at a given point of the observation plane consists of a superposition of a multitude of amplitude-spread functions, each arising from a different scattering point on the surface of the medium. Thus, the various spread functions sum up with markedly different phases, resulting in a highly complex pattern of interference, known as a laser speckle pattern. The formation of speckle patterns is a random process most comprehensively described by Goodman [1].

The speckle contrast parameter $K$ is defined as:

$$
K=\frac{\sigma}{\langle I\rangle}
$$

where $\sigma$ is the intensity standard deviation and $\langle\mathrm{I}\rangle$ is the mean intensity value. In the experiment, the local dynamics in scattering samples (moving scatterers) leads to speckle blurring due to the finite camera exposure time. This blurring, consequently, decreases the speckle contrast value that, in fact, allows quantifying the flow rate.

When the speckle patterns are observed with the finite integration time, $K$ is related to the electric field temporal autocorrelation function $G(\tau)=<E(t) E^{*}(t+\tau)>$. The measurable quantity $\mathrm{M}(\tau)$ can be linked to actual physical microscopic properties by the normalized field autocorrelation function $G(\tau)$ via the Siegert relation, $M(\tau)=1+\beta G(\tau)^{2}[2,3]$, with $M(\tau)=\frac{\langle I(t) I(t+\tau)\rangle}{\langle I(t)\rangle^{2}}$, and taking into account the dynamics of the scatter medium, the speckle contrast becomes:

$$
K=\left[\frac{2 \beta^{2}}{T} \int_{0}^{T}\left(1-\frac{t}{\tau}\right)\left[\frac{G(\tau)}{G(0)}\right]^{2} d \tau\right]^{1 / 2}
$$

Here, $\beta$ represents a multiplicative reduction in contrast which is not associated with the dynamics of the scattering particles, but related to the depolarization, coherence length, or mismatch between the image 
pixel and speckle size at the CCD camera. If the scattering objects are static, the field does not change and $K$ reduces to $\beta$. If the detected light is composed of dynamic and static components, $E(t)=E_{d}(t)+E_{s}$, the field autocorrelation function can be presented as $G(\tau)=(1-\rho)\left|G_{d}(\tau)\right|+\rho G(0)$, where $\rho=I_{S} /\left(I_{d}+I_{S}\right)$ is the part of detected light scattered at the static scattering particles.

The intensity autocorrelation function becomes $M(\tau)=1+\beta\left[(1-\rho)\left|G_{d}(\tau)\right|+\rho G(0)\right]^{2}$.

The temporal speckle contrast is calculated, but the ergodicity requirement is not fulfilled:

$$
K=\left[\frac{2 \beta^{2}}{T} \int_{0}^{T}\left(1-\frac{t}{\tau}\right)\left[\frac{(1-\rho)\left|G_{d}(\tau)\right|+\rho G(0)}{G(0)}\right]^{2} d \tau\right]^{1 / 2} .
$$

When the turbid medium is fully dynamic and there are no static scatterers $(\rho \rightarrow 1)$ and Eq.(3) simplifies to Eq.(2). However, if only static scatterers are presented $(\rho \rightarrow 0)$, Eq.(3) does not reduce to a constant speckle contrast value as one would expect for the speckle contrast represented by Eq.(1). Typically, in Laser Speckle Contrast Imaging (LSCI) the speckle contrast is estimated by spatial sampling, assuming that ergodicity is replaced ensemble sampling of speckles with a temporal sampling [4]. However, if any static scatterers are presented the requirement for this assumption is not fulfilled.

Introduced in 1981 [4], the LSCI technique is now a powerful cost-effective method allowing non-contact full-field real-time visualization of flows. A number of LSCI-based experimental systems have been successfully implemented in various applications, including cerebral blood flow monitoring [5], in vivo characterization of tumor and tumor vascular network [6], fundamental studies towards the ultimate understanding of the nature of 'biological zero' [7], investigation of the structural malformations of blood [8], assessment of influence of allergens on skin blood microcirculation [9], and other. Potentially LSCI can be also used for monitoring of various dynamic processes in liquids, including characterization of Brownian motion $[10,11]$ and turbulence $[12,13]$. 
A major limitation of LSCI for more advanced application in biomedicine is associated with the nonergodicity of the biological tissues. The biological tissues are heterogeneous composing both static and dynamic scattering particles/centers, and/or dynamic scattering areas overlaid by static scattering layers and vice versa. Therefore, averaging ensembles for time and space are different. The presence of static areas yields the non-ergodic speckle fluctuations that provide a systematic error for the quantitative interpretation of the images obtained.

The non-ergodic to ergodic transition has been studied by diffuse light scattering [14,15] and laser speckle imaging $[16,17]$ techniques. It has been shown $[14,17]$ that the ergodicity breaking is a signature of a transition towards an arrested state such as the aggregation process. Different approaches have been used to treat the problematic of non-ergodic highly scattering turbid medium for fifty years. The brute force method combining Monte Carlo modeling, multi-exposure speckle imaging (MESI) and spatial frequency domain imaging (SFDI) has been implemented [18,19]. Also, a dedicated experimental procedure with a dual laser source and multiple detectors allowing isolation of the single scattering contribution from the total scattered signal has been described [20-22]. Experimental procedures using an ergodic reference sample, for instance by slowly rotating the sample during the experiment [23], or using a second cell filled with dynamic material placed behind the sample (second cell randomizes and gently shakes the speckle pattern of the static sample) [24] are used to treat non-ergodicity. The last method indicates that ergodic and non-ergodic components co-exist in the data and can be separated for different calibration purposes.

In the experimental conditions, the duration of a single measurement, which is equivalent to the camera exposure time $T$, is much longer than the relaxation time of moving component of speckle pattern but much shorter than that of the static component. It is important to note that each raw image is an average signal collected during the exposure time. Thus, the definition of ergodicity and non-ergodicity depends on chosen camera exposure. Ergodicity in the general sense assumes temporal averaging during unlimited observation time. Nevertheless, the non-ergodicity caused by limited observation time can appear in the system that is ergodic in general sense. In particular, slow dynamics is ergodic process in general sense 
due to the equal temporal and spatial statistics during unlimited observation time. However, during the fixed exposure time the camera records the limited number of observed states that may turns the statistics to appear as non-ergodic [25]. Thus, in the experiment, the separation between ergodic and non-ergodic processes can be a complex task. However, it is possible to analyze the dynamics of speckle pattern for given exposure time to prevent systematic errors appearing with the presence of static elements in the system.

With the ultimate aim to understand the peculiarities of speckle patterns formation, in the current study we compare the spatial and temporal statistical approaches used for the LSCI analysis. A coefficient for characterization of contribution of static and dynamic components of the speckle pattern has been proposed. As an example of the systems with static/dynamic transitions, thawing and heating of the Intralipid samples were studied by LSCI approach. Thawing is a complex process of dramatic changes in dynamic properties of the scattering medium. During the thawing process, the considered system changes its state from fully static to dynamic via the intermediate mixed state. Heating of the liquid Intralipid sample expectedly leads to the enhancement of the Brownian motion. However, heating to the temperature of $45{ }^{\circ} \mathrm{C}$ and above leads to formation of a solid lipid film on top of the liquid layer representing the process of dynamic to static state transition. The introduced coefficient has been also used for characterization of dynamics changes in the tissue phantom with embedded capillary.

\section{Materials and methods}

\subsection{Experimental setup and scattering samples}

The classical LSCI setup has been used for measurements. The experimental setup utilizes $13 \mathrm{~mW}$ laser diode (RLD650-13-3, Roithner LaserTechnik GmbH, Austria) emitting the light at $655 \mathrm{~nm}$ that is further expanded by Thorlabs Engineered Diffuser (ED1-C20, Thorlabs, USA) providing the uniform angular distribution of the transmitted radiation. CMOS camera (DCC3240M, 1280×1024, pixel size $-6.7 \mu$ m, Thorlabs, USA) was used in combination with $12 \mathrm{~mm}$ F1.4 objective (Kenko Tokina Co., Ltd., Japan) for acquisition of gray-scale raw speckle images. The obtained images were processed by custom developed algorithm in offline regime using MATLAB r2017b software environment. 
Intralipid-5\% obtained by dilution of Intralipid-20\% (Intralipid®, Fresenius Kabi, Sweden) was used as a model fluid for monitoring of thawing and heating processes. Intralipid is an emulsion of soybean oil that is used widely for modelling of turbid scattering medium [26]. Petri dishes were used as phantom holders both for thawing and heating experiments.

\subsection{Methods of laser speckle images processing}

First and the most commonly used method of LSCI is the spatial analysis of speckle statistics using sliding window over raw speckle image obtained from CMOS or CCD camera [27]. Typically, the sliding window has the size of $5 \times 5$ or $7 \times 7$ pixels. However, in the result of spatial processing, the considered scheme has a disadvantage related to the loss of spatial resolution determined by the sliding window size. To avoid this problem an alternative method of temporal statistical analysis has been introduced for LSCI measurements [28]. The method implies the registration of several consecutive speckle patterns and the calculation of mean value and standard deviation of the time-integrated speckle intensity for each specific pixels of the pattern thus preserving the initial resolution. Both of the approaches have been utilized in the current study. Further, sSC and tSC abbreviation will be used for spatial and temporal speckle contrast respectively. For obtaining sSC images $7 \times 7$ pixels window has been used. For correct comparison, sequence of consecutive 49 frames has been used for obtaining tSC images. Each speckle image was recorded with $18 \mathrm{~ms}$ exposure. To reduce processing time the parallel computing has been implemented [29]. 

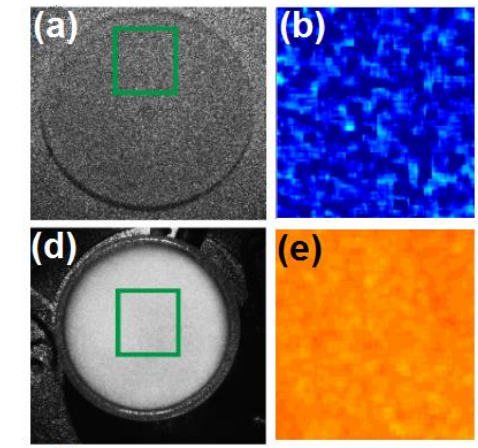

(e)
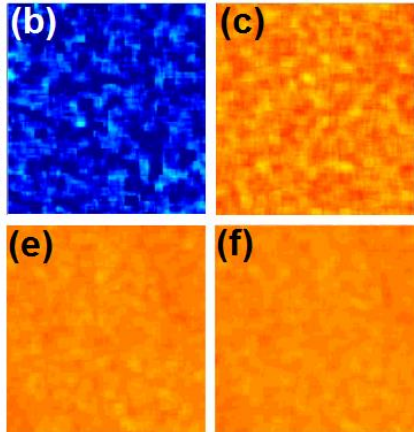

(f)
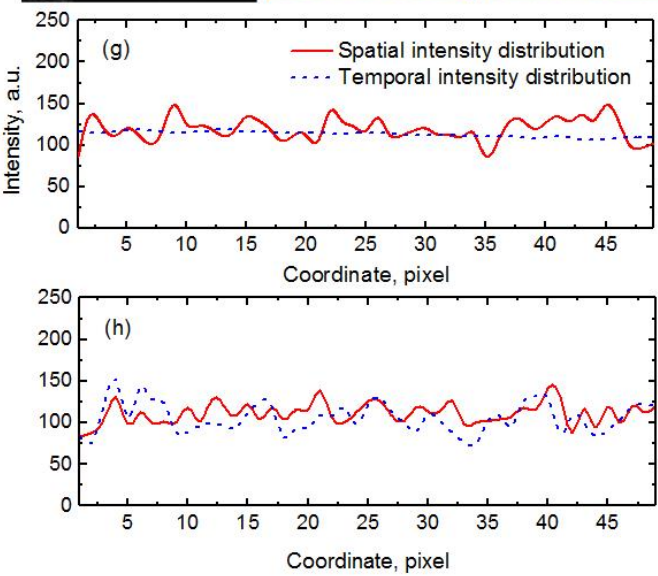

Figure 1. Raw speckle image for static scattering medium - ground glass diffusor placed on paper sheet (a), and corresponding images of sSC (b) and tSC (c); raw speckle image of dynamic scattering medium Intralipid solution in a Petri dish (d), and corresponding images of sSC (e) and tSC (f). Respectively, (g) spatial intensity distribution across the 49 pixels (solid line) and temporal intensity fluctuations, obtained at the same pixel of 49 consecutively measured laser speckle contrast images (dashed line), corresponding to (a-c); (h) spatial intensity distribution across 49 pixels (solid line) and temporal intensity fluctuations, obtained at the same pixel of 49 consecutively measured laser speckle contrast images (dashed line), corresponding to (d-f).

\subsection{Laser speckle contrast of dynamic and static systems}

In case of dynamic light scattering spatial and temporal approaches would give the same result. However, for the static areas, time and spatial averaging becomes not equal. For the ideal static speckle pattern, the theoretical speckle contrast value obtained by spatial processing should be equal to 1 . In this case, the dark and bright speckles take minimal and maximal intensity values correspondingly, thus, the mean 
intensity is equal to the standard deviation. Whereas, the speckle contrast obtained by the temporal processing approach in each particular pixel of the image should be equal to 0 , due to the fact that standard deviation over chosen image sequence tends to 0 for fully static speckle pattern. While, for dynamic speckle pattern, speckle contrast obtained by temporal processing should be greater than 0 due to the fact that standard deviation for dynamic speckle pattern cannot be equal to 0 . In the experiment it is impossible to achieve the ideal conditions, thus, 0 and 1 speckle contrast values usually cannot be obtained without additional post-processing. Nevertheless, the difference in results of static speckle pattern processing by spatial and temporal methods is significant in the experiments.

Figure 1 illustrates the difference between static and dynamic speckle patterns utilizing both, spatial and temporal processing approaches. In this experiment, round-shaped ground glass diffusor and the Intralipid 5\% solution poured into the Petri dish were used as the samples for generation of static and dynamic speckle patterns respectively.

Figure 1(a,d) shows the example of raw speckle images of diffusor and Petri dish obtained by the camera. Figure 1(b,e) shows sSC images in chosen ROI (Region of Interest) (green square on raw images). For the obtaining of the final sSC image, 49 raw frames were averaged. Figure 1(c,f) shows tSC image of the same ROI. As it mentioned previously, spatial processing reduces the resolution of the speckle contrast image. Thus, sSC and tSC images have different resolutions. So, for the correct comparison, the resolution of tSC images has been downgraded by averaging within $7 \times 7$ pixels sliding window.

It is clearly seen that sSC image of diffusor is trends to high values (blue color) that means the absence of any movements in ROI (figure 1-b). Some bright blue spots with low speckle contrast may appear on image because of significant roughness of the diffuser.

Figure 1-c shows tSC image of diffusor. As mentioned early (see Ref.[30]), speckle signals are uncorrelated in time and statistically independent for typical cameras exposure time. It is clearly seen that speckle contrast image trends to lower value (red color) corresponding to dynamic speckle pattern. 
Nevertheless, for Petri dish filled with Intralipid solution, the speckle contrast images obtained with both temporal and spatial processing give the same values (see figure 1-e and figure 1-f). To clearly demonstrate the difference between the spatial and temporal processing for the ergodic and non-ergodic systems, figure 1-g and figure 1-h shows spatial and temporal intensity distributions for randomly taken 49-pixel line and 49-value temporal sequence taken in one pixel for ground glass diffusor and 5\% Intralipid solution respectively. Thus, the described difference should be necessarily taken into account and compensated during data processing. Moreover, these differences between spatial and temporal processing can be used to distinguish static and dynamic areas during measurements with given exposure time. For this purposes we introduce the coefficient of speckle dynamics (CSD) as follow:

$$
C S D=\frac{2 K_{t}}{K_{s}+K_{t}}
$$

where $K_{S}$ is the speckle contrast over spatial window and $K_{t}$ is the speckle contrast over temporal window. Figure 2 shows the results of CSD calculations for the $K_{S}$ and $K_{t}$ presented in figure 1 both for static and dynamic scattering media. For the calculation of $K_{S}$ and $K_{t} 49$ consecutive raw frames were used. Firstly, as described above, 49 frames were used to obtain one $K_{t}$ image. Then, $49 K_{S}$ images has been calculated using $7 \times 7$ sliding window. Further, the obtained $K_{t}$ image has been used with each of $49 K_{S}$ images for calculation of 49 CSD maps. Finally, 49 CSD maps were averaged to obtain final CSD map. The higher values of CSD corresponds to the dynamic system while the lower values denoting the static areas (see Figure 2).

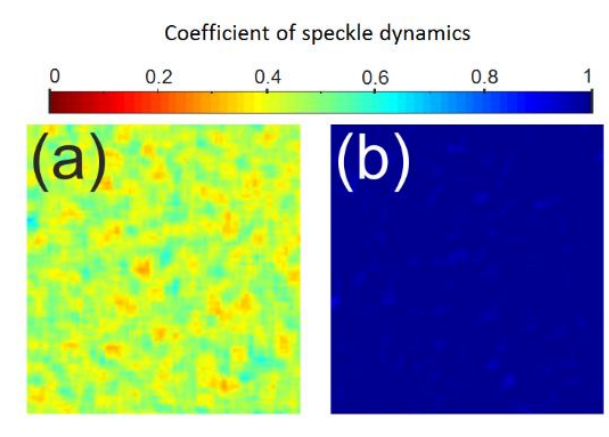

Figure 2 CSD map calculated using Eq. (4) for static (a) and dynamic (b) speckle patterns presented in Figure 1. 


\section{Results and Discussion}

\subsection{Heating experiment}
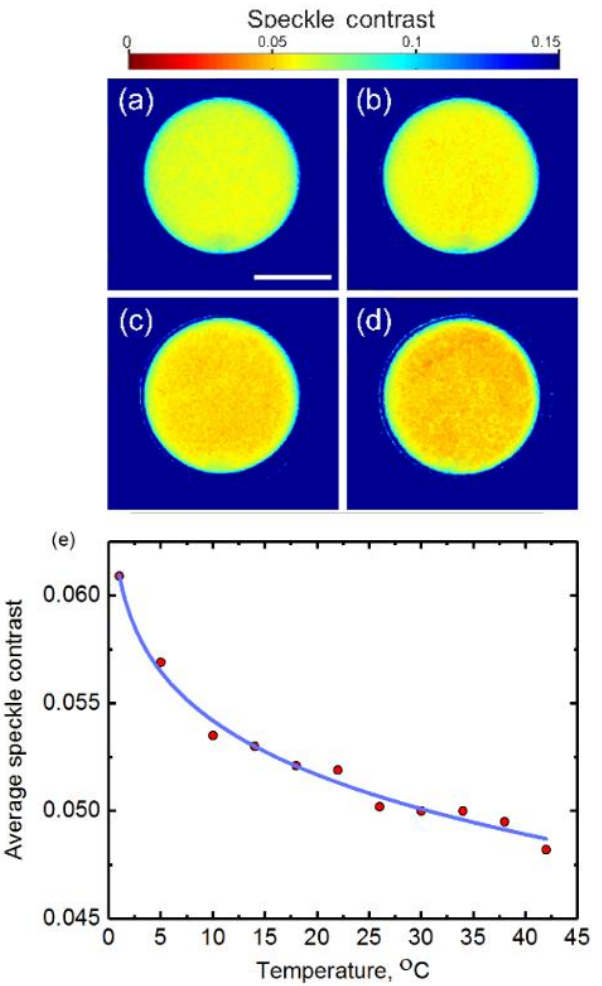

Figure $3 \mathrm{sSC}$ images of $5 \%$ Intralipid solution heating process for $1^{\circ} \mathrm{C}(\mathrm{a}), 5^{\circ} \mathrm{C}(\mathrm{b}), 14^{\circ} \mathrm{C}(\mathrm{c})$ and $26^{\circ} \mathrm{C}(\mathrm{d})$. Scale bar is equal to $10 \mathrm{~mm}$. Temperature dependence of the averaged speckle contrast for the heating process of Intralipid 5\% (red dots) and its fitting curve (blue line) (e).

To demonstrate the possibility of monitoring heating processes in liquids by LSCI technique the following experiment was performed: $3 \mathrm{ml}$ of $5 \%$ Intralipid were poured into a glass Petri dish and placed onto a computer-controlled cooling and heating thermo-plate (THMS600, Linkam, United Kingdom). The temperature of the liquid was remotely monitored with a thermal camera (FLIR, Sweden). Prior to the measurements, Intralipid was cooled to $1{ }^{\circ} \mathrm{C}$. After that the Intralipid sample was step-wise heated up to $42{ }^{\circ} \mathrm{C}$ with the step size of $3-5^{\circ} \mathrm{C}$. Figure 3 (a-d) shows the obtained speckle contrast images for the Intralipid- $5 \%$ at the temperature of $1,5,14$ and $26^{\circ} \mathrm{C}$, respectively. From this figure it is clearly seen that the speckle contrast decreases as the temperature grows. This is related to the intensification of the 
movement during the sample heating. Figure 3-e shows the results of the temperature-depended measurements of the speckle contrast averaged over the surface area of the entire Petri dish. Here, one can see the continuous decrease of the spatially averaged speckle contrast during heating due to the increase of the Brownian motion speed with the temperature rise.
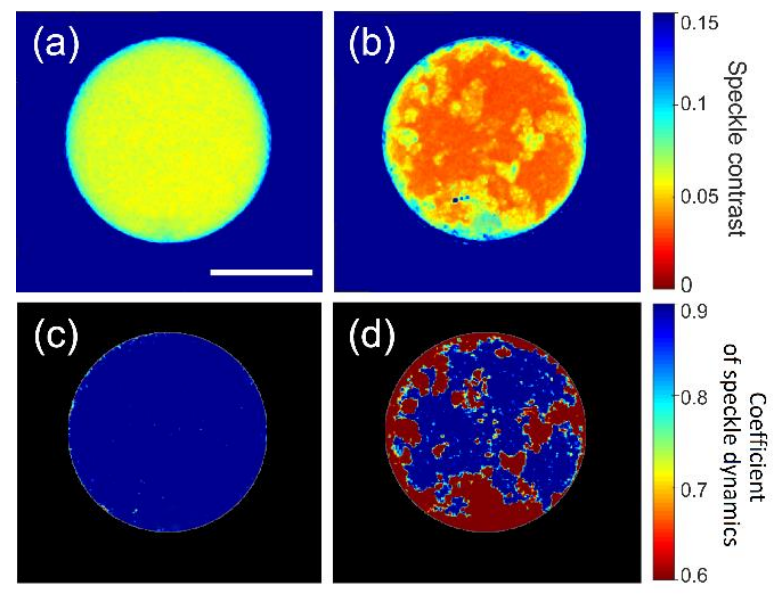

Figure 4. $\mathrm{sSC}$ images of $5 \%$ Intralipid solution at $1^{\circ} \mathrm{C}$ (a) and $53^{\circ} \mathrm{C}(\mathrm{b})$. Scale bar is equal to $10 \mathrm{~mm}$. Solid lipid film formed at $53{ }^{\circ} \mathrm{C}$ is partly removed. (c) and (d) - the corresponding CSD maps calculated using Eq.(4).

Moreover, it was discovered that a solid lipid film on the Intralipid surface could appear during heating to the temperature of $45^{\circ} \mathrm{C}$ and above. Thus, the Intralipid heating process could be considered as the process with dynamic to static transition. Figure $4(a, b)$ shows a comparison of speckle contrast images for $5 \%$ Intralipid solution at $1{ }^{\circ} \mathrm{C}$ and $53^{\circ} \mathrm{C}$. To observe the difference, some parts of the lipid film formed at $53^{\circ} \mathrm{C}$ were gently removed with the syringe needle (see Figure 4-b). Thus, the considered sample represents the mixture of solid and liquid areas. The areas with high values of speckle contrast in the Figure 4-b correspond to decreasing of dynamics. In fact, for the complex heterogeneous scattering medium containing static and non-static inclusions the interpretation of changes of speckle contrast is often more complicated. For example, it's not nessecerally that only changes in flow or dynamics of scattering particles can influence the speckle contrast, it also can be changed by roughness of the surface or bottom of the samples [31] or their movement artifacts [32]. In this point of view, the CSD mapping can be more accurate for assessment of speckle images. So, the CSD naps was calculated for the considered experiment (see Figure 4(c,d)). As it mentioned previously, the higher values of the CSD 
corresponding to the dynamic system are shown with blue color and the lower values denoting the static areas are shown with dark red color. Area outside of ROI has been colored in black for the better clarity. From figure 4-c it is clearly seen that the liquid Intralipid sample at $1^{\circ} \mathrm{C}$ represents fully dynamic system. However, the appeared solid lipid film (see Figure 4-d) turns the considered system to the static state. It is clearly seen that the areas corresponding to the solid film pieces indicate static areas.

Thus, calculation of CSD by the proposed method can be useful to distinguish real changes in motion from appearance of static components in the field of view.

\subsection{Thawing experiment}

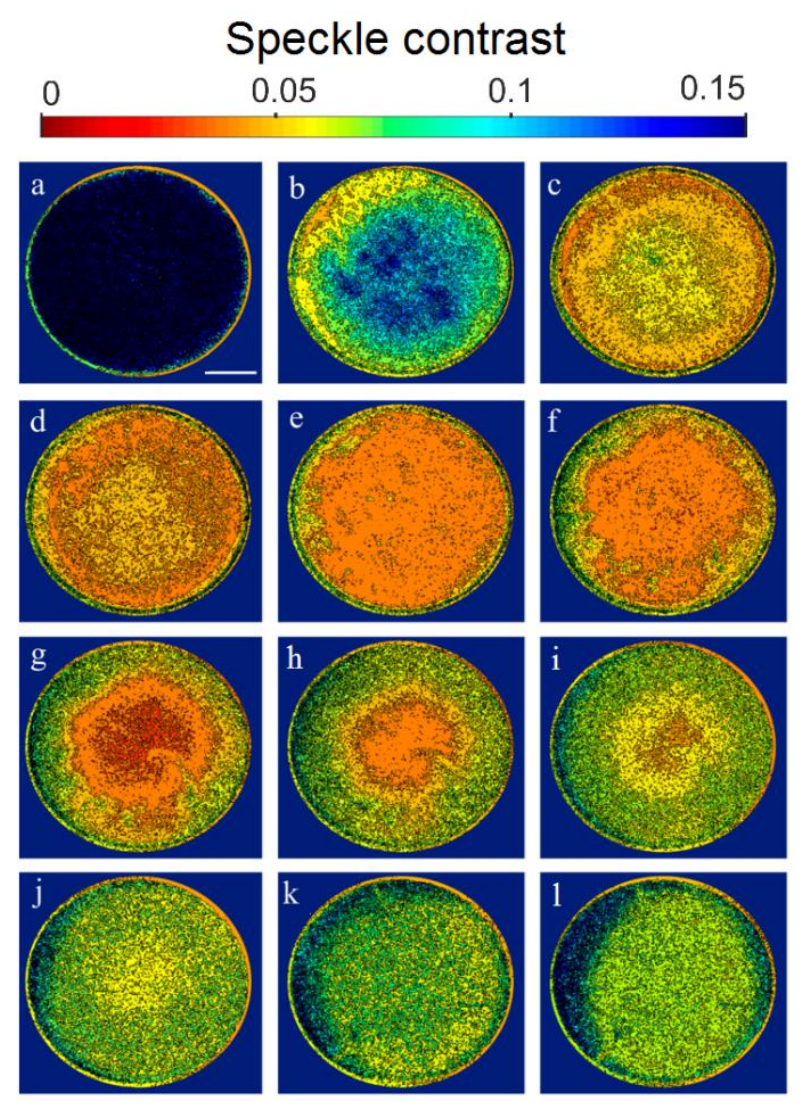

Figure 5. sSC images of the Intralipid solution thawing process for 0 (a), 5 (b), 10 (c), 15 (d), 20 (e), 30 (f), 40 (g), 50 (h), 60 (i), 70 (j), 120 (k) and 180 (1) min after starting the experiment. Scale bar is equal to $10 \mathrm{~mm}$. 
To demonstrate the possibility of monitoring the complex processes with a transition from the static to dynamic state (including the combinations of these states) by LSCI technique, we have performed the following experiment: $5 \mathrm{ml}$ of $5 \%$ Intralipid solution was poured into a Petri dish and placed into a refrigerator at $-19{ }^{\circ} \mathrm{C}$ until complete freezing. The Petri dish with the frozen Intralipid was then placed onto an air damped optical table (Newport Inc., USA) to prevent possible external vibrations for the imaging with the LSCI system mounted on the same table. The thawing process of the Intralipid solution was monitored during 3 hours at the room temperature $\left(21^{\circ} \mathrm{C}\right)$. The 50 consecutive raw speckle images were recorded each minute for the processing.

Figure 5 displays the sSC images for the selected moments of time during the thawing process. As one can see, at the beginning of experiment, speckle contrast has a maximum value. This is due to absence of scattering particles motion for the given exposure time when the Intralipid sample is frozen. In about 5 minutes time due to thawing of Intralipid sample along the edge of the Petri dish the speckle contrast in this area is decreasing (see Figure 5-b and Figure 5-c). In 15 minutes the speckle contrast is dramatically decreased over the whole Petri dish (see Figure 5-d). This is associated with covering of the surface of the frozen sample (static area) by thin layer of thawed Intralipid solution. The further evolution of speckle contrast is observed during the next 60 minutes (see Figure 5 (e-i)) correspondingly to the complete melting of the frozen area under the liquid layer, and an increase of the speckle contrast values (see Figure $5(\mathrm{j}-1)$ ) indicates that thawing process is completed and the speckles are defined only by Brownian motion.

Figure 6 shows the normalized tSC and sSC values averaged over the sample area and averaged CSD for the first 30 minutes of the experiment. As one can see, during the first 7 minutes tSC and sSC take different values due to the systematic error of temporal processing appearing with the presence of static area. Further, after $10-15$ minutes from the start of the experiment the deviations of the temporal and spatial speckle contrasts become saturated. Also, it is seen that after 7 min the CSD became saturated that corresponds to the dynamic state when the averaged speckle contrast values obtained with temporal and spatial processing are very close. As it was mentioned previously, static frozen area can be covered by 
dynamic liquid layer. After 7 min from start of experiment the thickness of this layer is enough to count whole system as dynamic. In this point of view, dynamic layer appearing during thawing can be considered similar to an ergodic reference sample, as described in Ref. [23,24] and allowing us to use the temporal processing providing a higher resolution without errors related to presence of static components.

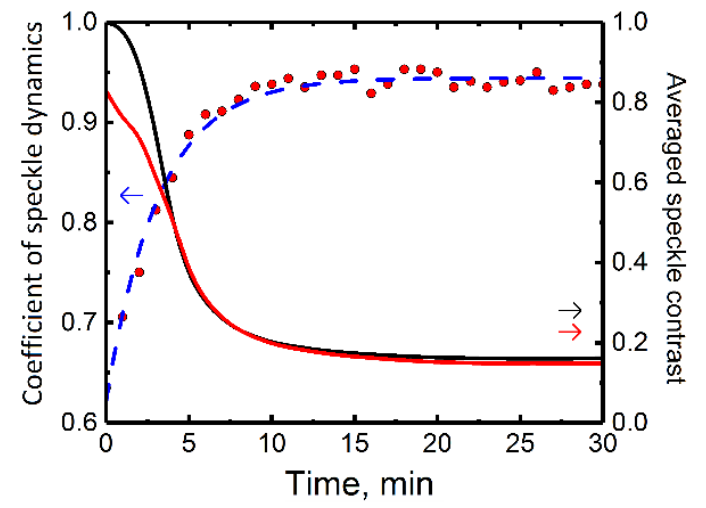

Figure 6 Evolution of speckle contrast during the thawing of Intralipid solution: black and red lines represent, respectively, relative values of normalized spatial and temporal speckle contrasts in comparison with the CSD measured experimentally (red dots); dashed line represents fitting curve.

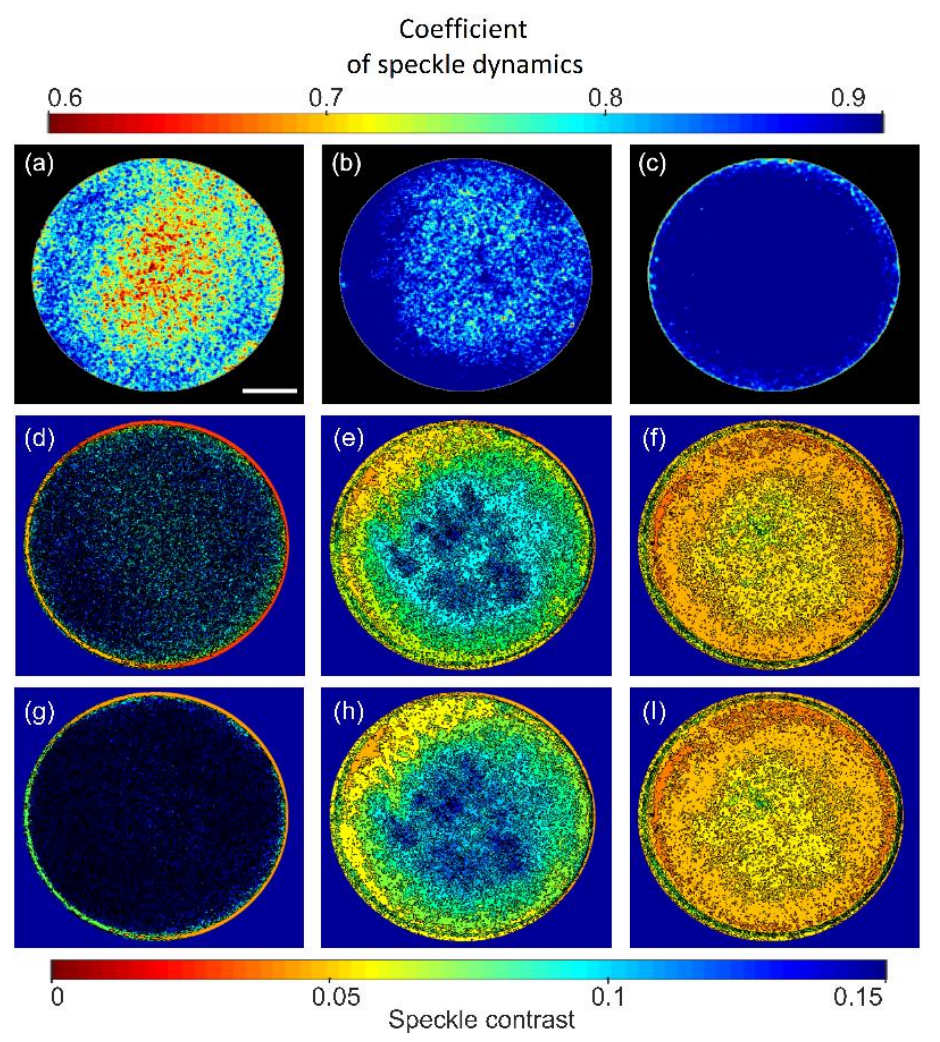


Figure 7 CSD maps of the Intralipid solution thawing process obtained after 1 (a), 5 (b) and 10 (c) minutes and corresponding tSC (d,e and $\mathrm{f}$ ) and $\mathrm{sSC}(\mathrm{g}, \mathrm{h}$ and i) images. Scale bar is equal to $7 \mathrm{~mm}$.

Figure 7 shows the CSD, maps calculated for the thawing process at 1 (see Figure 7-a), 5 (see Figure 7-b) and 10 (see Figure 7-c) minutes after the beginning of the experiment. In this experiment, 49 consecutive raw frames have been recorded for calculation of $K_{S}$ and $K t$. Thus, 49 CSD maps have been averaged to obtain final CSD map. Important to notice that difference between SSC and tSC images can be seen for the thawing process at 1 minute after the beginning of the experiment (see Figures 8-d and 8-g). Some differences can be seen between sSC and tSC images after 5 minutes from beginning of the experiment (see Figures 8-e and 8-h) while no significant differences is noticeable after 10 minutes (see Figures 8-f and 8-i). Also, it is clearly seen that the sample gradually becomes dynamic along the edge of the Petri dish to the inner part during the first few minutes after experiment starting. In a 7 minutes time till the end of the experiment the whole sample in the Petri dish is observed to be dynamic.

Thus, utilizing LSCI approach describing above it is possible to identify and quantitatively assess the stages of thawing phases, peculiar properties of the dynamic processes and transition from static to dynamic stage.

\subsection{Tissue phantom experiment}

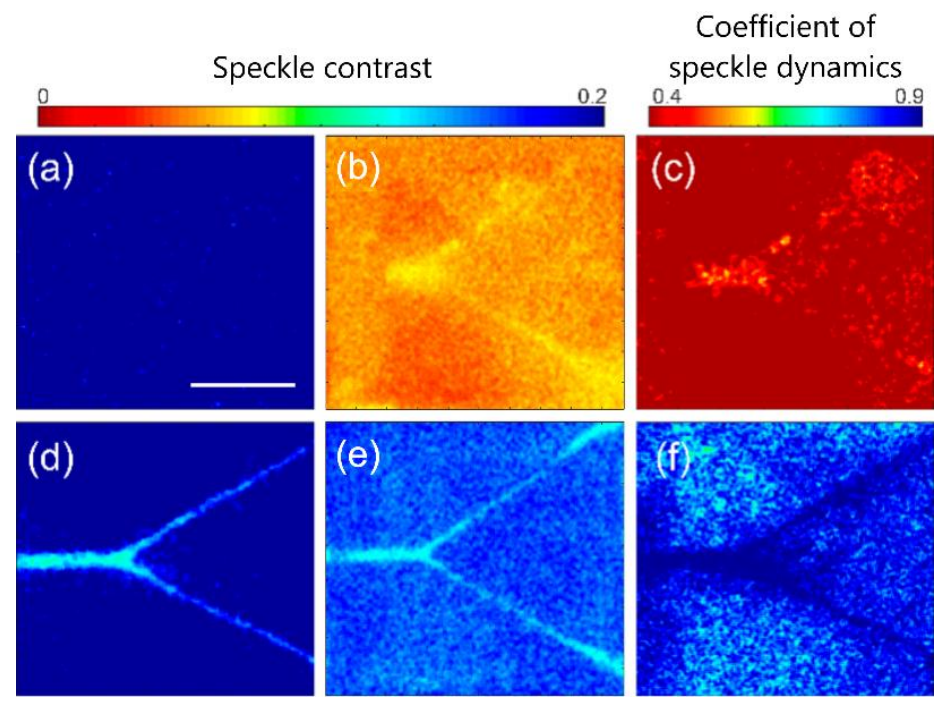

Figure 8. sSC maps for the tissue phantom with no flow (a) and for the flow rate of $2 \mathrm{ml} / \mathrm{min}$ (d). tSC maps for the phantom with no flow (b) and the flow of $2 \mathrm{ml} / \mathrm{min}$ (e). CSD maps for the phantom with no flow (c) and the flow of $2 \mathrm{ml} / \mathrm{min}$ (f). Scale bar is equal to $7 \mathrm{~mm}$. 
Figure 8 shows sSC, tSC and CSD maps for the experiment with the tissue phantom containing a Yshaped hollow channel with the diameter of $1 \mathrm{~mm}$. The phantom is made of PVC [33,34] and has the absorption and scattering coefficients of $0.07 \mathrm{~mm}^{-1}$ and $1 \mathrm{~mm}^{-1}$ respectively. The embedding depth of the channel is about $2 \mathrm{~mm}$. Intralipid 5\% was pumped with the syringe pump (Fusion 100, Chemix, United States) at the rate of $2 \mathrm{ml} / \mathrm{min}$ through the phantom channel. In this experiment, 49 consecutive raw frames have been recorded for calculation of $K s$ and $K t$. Thus, 49 CSD maps have been averaged to obtain final CSD map. It is clearly seen that in the absence of any motion the sSC and tSC maps are different (see Figures 8-a and 8-b). The CSD map (see Figure 8-c) in this case shows the low values as it is a fully static system. Important to note that in the presence of flow the spatial and temporal speckle contrast inside the vessel takes equal values while the speckle contrast values for area outside the vessel are different. However, this difference is smaller than for the static case (see Figures 8-d, 8-e and 8-a, 8b). This effect can be explained by the fact that the movement inside the channel can cause the vibration of whole phantom structure that subsequently leads to the reduction of speckle contrast. Therefore, the area outside the vessels became not fully static that is clearly seen from corresponding CSD map (see Figure 8-f). Thus, CSD mapping can be a possible marker for detection of micro movements during the measurements.

For more advanced flow and dynamics assessment, the proposed method can be used combined with multi-exposure speckle contrast imaging [35], optical clearing [36], etc. Moreover, it should be emphasized that the approach, described above, can be used not only in biological studies, but can find straightforward industrial applications. More specifically, it is very likely that CSD mapping can complement conventional speckle imaging approach in paint drying monitoring and/or roughness measurements $[37,38]$.

\section{Conclusions}

We examined LSCI at the conditions of dynamic/static state transition with a final aim of assessment of limitations in quantitative interpretation of speckle contrast images. Based on the simple phenomenological approach we introduced the coefficient of speckle dynamics to assess quantitatively 
fraction of the dynamic part of scattering medium in the static one and for separating static and dynamic speckle patterns for given exposure time with aim to distinguish real changes in motion from appearance of static components in the field of view. We showed that presence of dynamic layer above the static part of the sample allows to neglect all limitations of spatial processing of laser speckle contrast images. This result is in an excellent agreement with the results of alternative studies performed early. Also, we showed that proposed method can be used for assessment of the flow changes in the tissue phantom. High flow rates inside phantom vessels can lead to vibration of the phantom structure which can be detected and estimated by proposed method. The presented experimental findings have a strong potential to be used in future practical applications of LSCI to distinguish between the static parts of scattering medium, surface roughness variations and the dynamic areas attributed to the flows. The introduced coefficient allows quantification of the eligibility conditions when the spatial approach of speckle pattern processing can be replaced with more preferable time processing preserving high spatial resolution. Furthermore, the introduced speckle dynamics coefficient can be potentially find an application in the evaluation of vessels masking and very likely for estimation of biological tissue micro-vibrations.

\section{Disclosures}

The presenting authors do not have any financial conflicts of interest regarding the content of this manuscript.

\section{Acknowledgments}

This work was supported by the CIMO Fellowships program (21.3.16/TM-16-10089/CIMO Fellowship/WS19), EDUFI Fellowship program (13.10.17/TM-17-10655/EDUFI Fellowship/ WS 19), the Academy of Finland (Grant No. 290596), the Tomsk State University Academic D I Mendeleev Fund Program and the National Research Nuclear University MEPhI's Academic Excellence Project (Contract No. 02.a03.21.0005). The authors are grateful to A Dombovari, MSc, and Dr G Lorite for their help in the preparation of samples and their useful discussions. 


\section{References}

[1] W. Goodman, "Statistical properties of laser speckle patterns," in: Laser speckle and related phenomena (Springer Berlin Heidelberg, 1975).

[2] J.D. Briers, A.F. Fercher, "Laser Speckle Technique for the Visualization of Retinal Blood Flow,” Proc. SPIE 369, 22-28 (1983).

[3] R. Bandyopadhyay, A.S. Gittings, S.S. Suh, P.K. Dixon, and D.J. Durian, "Specklevisibility spectroscopy: A tool to study time-varying dynamics,” Rev. Sci. Instruments 76 (9), $093110(2005)$

[4] A.F. Fercher, and J.D. Briers, "Flow visualization by means of single-exposure speckle photography," Opt. Commun. 37(5), 326-330 (1981).

[5] V. Kalchenko, D. Israeli, Y.L. Kuznetsov, I. Meglinski, and A. Harmelin, "A simple approach for non-invasive transcranial optical vascular imaging (nTOVI)", J. Biophoton. 8(11-12), $897-901$ (2015).

[6] V. Kalchenko, N. Madar, I. Meglinski, and A. Harmelin, "In vivo characterization of tumour and tumour vascular network using a multi-mode imaging approach”, J. Biophoton. 4(9), 645-649 (2011).

[7] I. Meglinski, V. Kalchenko, Y. Kuznetsov, B. Kuznik, and V.V. Tuchin, "Towards the nature of biological zero in the dynamic light scattering diagnostic techniques", Doklady Physics, 58(8), 323 - 326 (2013).

[8] A.N. Korolevich, I. Meglinski, "The experimental study of the potentialities of diffusing wave spectroscopy for the investigating of the structural characteristics of blood under multiple scattering”, Bioelectrochemistry, 52(2), 223-227 (2000).

[9] V. Kalchenko, Y.L. Kuznetsov, D. Prise, I. Meglinski, and A. Harmelin, "Mouse ear swelling test for weak allergens by using laser speckle imaging with the long exposure time," J. Biomed. Opt. 19(6), 60502 (2014). 
[10] G.Volpe, G. Volpe, and S. Gigan, "Brownian motion in a speckle light field: tunable anomalous diffusion and selective optical manipulation," Scientific Reports 4, 3936 (2014).

[11] D.A. Boas, I. Meglinski, L. Zemany, L. E. Campbell, B. Chance, and A. G. Yodh, "Diffusion of Temporal Field Correlation with Selected Applications," in CIS Selected Papers: Coherence-Domain Methods in Biomedical Optics (Edited by V.V. Tuchin, Bellingham, Washington: SPIE Press, 1996), Vol. 2732.

[12] I.V. Meglinski, D.A. Boas, L. E. Campbell, A. G. Yodh, and B. Chance, "Diffuse photon probes as a tool for diagnostic of laminar and turbulent flow localized in highly scattering inhomogeneous media," in Annual Retreat of the Department of Biochemistry and Biophysics (Swarthmore College, Philadelphia, PA, USA, 19 February, 1995).

[13] N.A. Fomin, Speckle photography for fluid mechanics measurements (Springer Science \& Business Media, 2013).

[14] B. Ruzicka, L. Zulian, and G. Ruocco, "Ergodic to non-ergodic transition in low concentration Laponite", J. Phys.: Condens. Matter. 16, S4993 (2004).

[15] M. Manno, D. Bulone, V. Martorana, and P.L. San Biagio, "Ergodic to non-ergodic transition monitored by scattered light intensity statistics," Phys. A. 341, 40-54 (2004).

[16] P. Zakharov, "Ergodic and non-ergodic regimes in temporal laser speckle imaging," Opt.Lett. 42(12), 2299 (2017)

[17] P. Zakharov, A. C. Völker, F. Buck, B. Weber, and F. Scheffold, "Non-ergodicity correction in laser speckle biomedical imaging," Proc. SPIE 6631 (2007).

[18] P. Zakharov, A. Völker, A. Buck, B. Weber, and F. Scheffold, "Quantitative modeling of laser speckle imaging," Opt. Lett. 31(23), 3465-67 (2006).

[19] T.B. Rice, E. Kwan, C.K. Hayakawa, A.J. Durkin, B. Choi, and B.J. Tromberg, "Quantitative, depth-resolved determination of particle motion using multi-exposure, spatial frequency domain laser speckle imaging," Biomed. Opt. Express 4(12), 2880-2892 (2013). 
[20] E. Overbeck and C. Sinn, "Three-dimensional dynamic light scattering," J. Mod. Opt. 46(2), 303-326 (1999).

[21] K. Schatzel, "Suppression of multiple scattering by photon cross-correlations techniques," J. Mod. Opt. 38(9), 1849-1865 (1991).

[22] C.G. de Kruif, J.K.G. Dhont, and H.J. Mos, "Multiple light scattering in colloidal suspensions: cross-correaltions photon counting spectroscopy,” J. de Phys. 46(3), 149-163 (1985).

[23] M. Medebach, N. Freiberger, and O. Glatter, "Dynamic light scattering in turbid nonergodic media.” Rev. Sci. Instr. 79, 073907 (2008).

[24] C. Haro-Pérez, G.J. Ojeda-Mendoza, and L.F. Rojas-Ochoa, "Three dimensional crosscorrelation dynamic light scattering by non-ergodic turbid media." J. Chem. Phys. 134, $244902(2011)$

[25] P.N. Pusey, and W.V. Megen, "Dynamic light scattering by non-ergodic media," Phys. A 157(2), 705 (1989).

[26] S.T. Flock, S.L. Jacques, B.C. Wilson, W.M. Star, and M.J. van Gemert, "Optical properties of Intralipid: a phantom medium for light propagation studies,” Lasers Surg. and Med. 12(5), 510-519 (1992).

[27] P. Zakharov, F. Scheffold, and B. Weber, "Laser Speckle Imaging of Cerebral Blood Flow," in Optical Imaging of Neocortical Dynamics (Edited by B. Weber, F. Helmchen, Springer Berlin Heidelberg, 2014), Vol. 85.

[28] H. Cheng, Q. Luo, S. Zeng, S. Chen, J. Cen, and H. Gong, "Modified laser speckle imaging method with improved spatial resolution,” J. Biomed. Opt. 8(3), 559-564 (2003).

[29] W.J. Tom, A. Ponticorvo, and A.K. Dunn, "Efficient processing of laser speckle contrast images,” IEEE Trans. Med. Im. 27(12), 1728-1738 (2008).

[30] H. Cheng, Y. Yan and T.Q. Duong, "Temporal statistical analysis of laser speckle images and its application to retinal blood-flow imaging," Opt. Exp. 16(14), 10214 (2008). 
[31] L. Tchvialeva, I. Markhvida, H. Zeng, D. I. McLean, H. Lui, H., and T. K. Lee, "Surface roughness measurement by speckle contrast under the illumination of light with arbitrary spectral profile,” Opt.Las.Eng., 48(7-8), 774-778 (2010).

[32] G. Mahé, P. Rousseau, S. Durand, S. Bricq, G. Leftheriotis, and P. Abraham, "Laser speckle contrast imaging accurately measures blood flow over moving skin surfaces," Microvasc. res., 81(2), 183-188 (2011).

[33] M.S. Wróbel, A.P. Popov, A.V. Bykov, M. Kinnunen, M. Jędrzejewska-Szczerskaa, and V.V. Tuchin, "Measurements of fundamental properties of human skin phantoms," J. Biomed. Opt., 20(4), 045004, 1-10 (2015).

[34] A.V. Bykov, A.P. Popov, A.V. Priezzhev, and R. Myllyla, "Multilayer tissue phantoms with embedded capillary system for OCT and DOCT imaging,” Proc. SPIE, 8091, 80911R (2011)

[35] L. M. Richards, S.S. Kazmi, K.E. Olin, J.S. Waldron, D. J. Fox Jr, and A. K. Dunn, "Intraoperative multi-exposure speckle imaging of cerebral blood flow," J. Cereb. Blood Flow Metab. 37(9), 3097-3109 (2017).

[36] A. Yu. Sdobnov, M. E. Darvin, E. A. Genina, A. N. Bashkatov, J. Lademann, and V. V. Tuchin, "Recent progress in tissue optical clearing for spectroscopic application," Spectrochim. Acta Part A: Mol. Biomol. Spectrosc. (2018). https://doi.org/10.1016/j.saa.2018.01.085

[37] L.C. Leonard, nd V. Toal, "Roughness measurement of metallic surfaces based on the laser speckle contrast method," Optics and Lasers in Engineering, 30(5), 433-440 (1998)..

[38] R. Arizaga, E.E. Grumel, N. Cap, M. Trivi, J.I. Amalvy, B. Yepes, and G. Ricaurte,. "Following the drying of spray paints using space and time contrast of dynamic speckle," JCT research, 3(4), 295-299 (2006). 